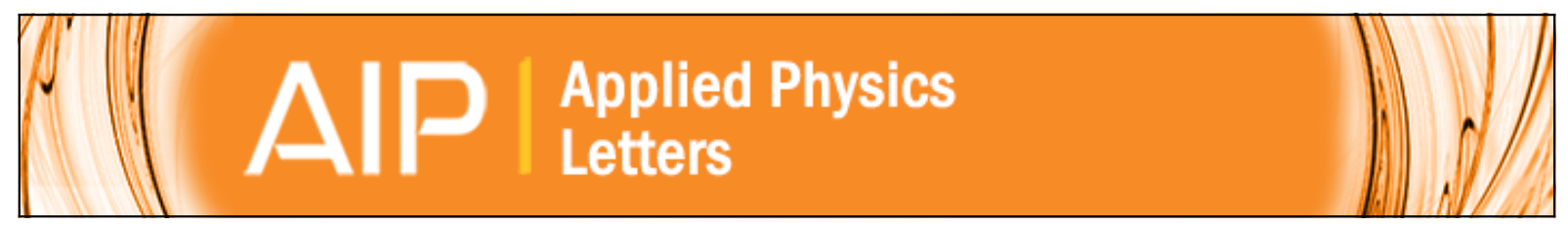

\title{
Photoconductivity from PbS-nanocrysta/semiconducting polymer composites for solution-processible, quantum-size tunableinfrared photodetectors
}

S. A. McDonald, P. W. Cyr, L. Levina, and E. H. Sargent

Citation: Applied Physics Letters 85, 2089 (2004); doi: 10.1063/1.1792380

View online: http://dx.doi.org/10.1063/1.1792380

View Table of Contents: http://scitation.aip.org/content/aip/journal/apl/85/11?ver=pdfcov

Published by the AIP Publishing

\section{Articles you may be interested in}

Charge separation dynamics at bulk heterojunctions between poly(3-hexylthiophene) and $\mathrm{PbS}$ quantum dots J. Appl. Phys. 118, 055502 (2015); 10.1063/1.4926869

Viability of using near infrared $\mathrm{PbS}$ quantum dots as active materials in luminescent solar concentrators Appl. Phys. Lett. 96, 191901 (2010); 10.1063/1.3422485

Steady state photoinduced absorption of $\mathrm{PbS}$ quantum dots film Appl. Phys. Lett. 92, 141108 (2008); 10.1063/1.2907492

Enhanced infrared photovoltaic efficiency in PbS nanocrystal/semiconducting polymer composites: 600-fold increase in maximum power output via control of the ligand barrier

Appl. Phys. Lett. 87, 233101 (2005); 10.1063/1.2137895

Size-tunable infrared (1000-1600 nm) electroluminescence from PbS quantum-dot nanocrystals in a semiconducting polymer

Appl. Phys. Lett. 82, 2895 (2003); 10.1063/1.1570940

\section{AIP $\left.\right|_{\text {APL Photonics }}$ \\ APL Photonics is pleased to announce Benjamin Eggleton as its Editor-in-Chief}

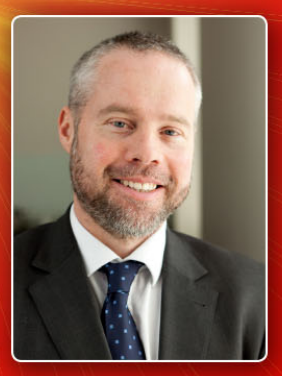




\title{
Photoconductivity from PbS-nanocrystal/semiconducting polymer composites for solution-processible, quantum-size tunable infrared photodetectors
}

\author{
S. A. McDonald, ${ }^{\text {a) }}$ P. W. Cyr, L. Levina, and E. H. Sargent \\ Department of Electrical \& Computer Engineering, University of Toronto, 10 King's College Road, Toronto, \\ Ontario, M5S 3G4, Canada
}

(Received 21 April 2004; accepted 13 July 2004)

\begin{abstract}
We report photoconductivity at infrared wavelengths, $975-1300 \mathrm{~nm}$, from a polymer/nanocrystal quantum dot composite. Biased films of the conjugated polymer poly[2-methoxy-5( 2 '-ethylhexyloxy-p-phenylenevinylene)] (MEH-PPV) sensitized with $\mathrm{PbS}$ nanocrystals $(\sim 5 \mathrm{~nm}$ diameter) demonstrate photocurrent at wavelengths beyond the response of the polymer and corresponding to the absorption of the nanocrystals. The photocurrent is attributed to absorption in the nanocrystals with subsequent hole transfer to the polymer and had an internal quantum efficiency of $\sim 5 \times 10^{-6}$ to $\sim 10^{-5}$ charges/photon at $5 \mathrm{~V}$ bias. (C) 2004 American Institute of Physics. [DOI: 10.1063/1.1792380]
\end{abstract}

In semiconductor nanocrystal (NC) quantum dots, the confinement of charge carriers to length scales less than the exciton-Bohr radius allows investigation and exploitation of material behavior in the size regime between the atomic and bulk. Tunability of the NC band gap via the quantum size effect has attracted attention for visible and infrared light emitting diodes, ${ }^{1-4}$ visible lasers, ${ }^{5,6}$ visible and infrared modulators, ${ }^{7-9}$ and visible photovoltaics. ${ }^{10}$

The use of NCs size-tuned to provide absorption onsets in the infrared is appealing for applications in sensing, night vision, biomedical assays in the second water window, ${ }^{11}$ and telecommunications. In the present work, we demonstrate photoconductivity in the infrared using a nanocomposite of $\mathrm{PbS}-\mathrm{NCs}$ in poly[2-methoxy-5-(2'-ethylhexyloxy$p$-phenylenevinylene)] (MEH-PPV). The resulting ability to detect light in the infrared using processible, physically flexible materials would be attractive for large-area, low-cost detector-based device realization, especially when compared to the lattice-matched, rigid-substrate, epitaxial growth requirements imposed by traditional III-V semiconductorbased detectors.

To date, work on photoconductivity in such nanocomposites has focused on cadmium chalcogenide nanocrystals, which absorb in the visible region. Wang and Herron reported the first demonstration of photoconductivity in a polymer doped with semiconductor nanoclusters (CdS) in 1992. ${ }^{12}$ Since then, others have studied charge separation in polymer/NC composites using $\mathrm{Cd}$ chalcogenide $\mathrm{NCs}{ }^{13,14}$ Studies of photoconductivity in pure CdSe-NC films have also been reported in both sandwich and lateral structures. ${ }^{15-17}$ Visible photoconduction in PbS in a purely inorganic system ${ }^{18}$ and the photorefractive properties of bulk-like PbS crystals studied in the infrared ${ }^{19}$ have also been reported. The present work demonstrates infrared photoconductivity from a solution-processed polymer/NC composite.

Compared with Cd-based chalcogenides, $\mathrm{PbS}$ presents a challenge in seeking to achieve exciton dissociation in a

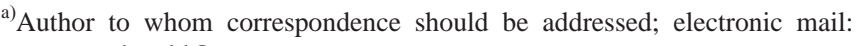
steve.mcdonald@utoronto.ca
polymer/NC composite. Since conjugated polymers typically have better hole than electron mobility, photoconductivity in polymer/NC composites mandates energy alignments that favor transfer of the photogenerated hole to the polymer. This requires that the highest occupied molecular orbital (HOMO) level of the polymer lie closer to vacuum than the valence band of the NC. The Cd chalcogenides have bulk ionization potentials between $\sim 6.4$ and $\sim 7.3 \mathrm{eV}$, while that of $\mathrm{PbS}$ is $\sim 4.95 \mathrm{eV}$. A variety of polymers are readily available with HOMO levels that provide the correct band alignment with $\mathrm{Cd}$ chalcogenides. However, the low ionization potential of $\mathrm{PbS}$ limits the number of readily available conjugated polymers that provide a favorable energy alignment. The polymer used herein is MEH-PPV (Fig. 1 inset), which has a HOMO level variously reported between $\sim 4.9$ and $\sim 5.1 \mathrm{eV} .^{20,21}$ Whether this polymer forms a type-I or type-II heterostructure with PbS-NCs is unknown due to the uncertainty in the vacuum-referenced band edges of both the organic and inorganic component (see the inset to Fig. 3). Furthermore, the possible formation of a dipole layer at the interface, which would change the effective band alignment, and the variation in energy levels with NC size add complications to the de-

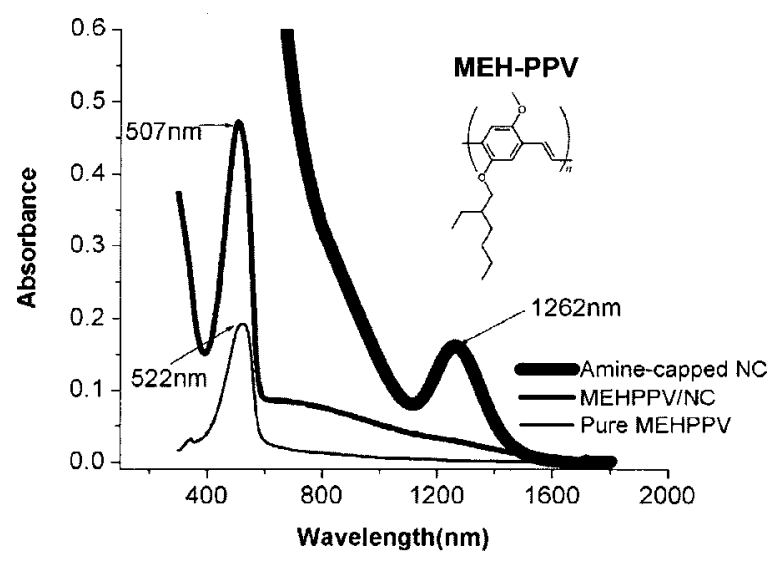

FIG. 1. Absorbance spectra for octylamine-capped PbS-NC in solution (thick weight, upper), MEH-PPV/PbS-NC blend on ITO (medium weight, middle), and pure MEH-PPV on ITO (thin weight, lower). The inset shows the chemical structure of MEH-PPV. 


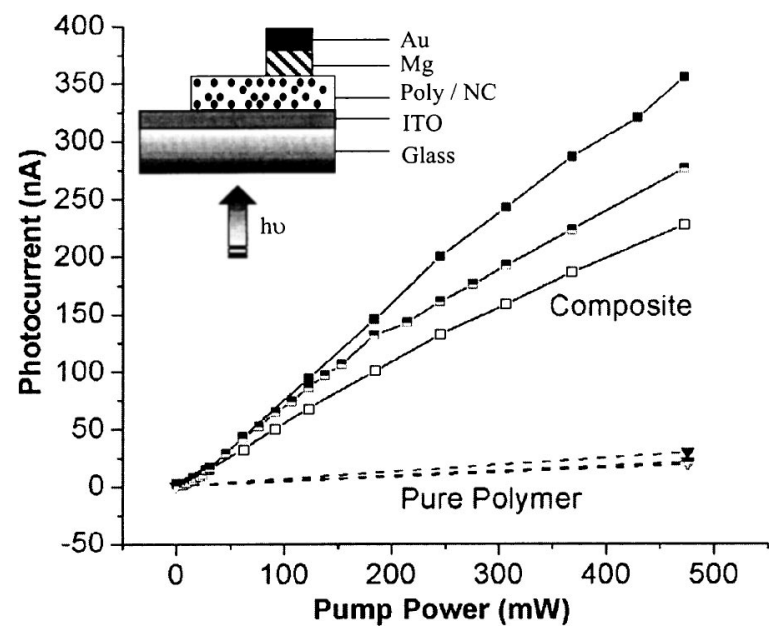

FIG. 2. Photocurrent as a function of pump power at $5 \mathrm{~V}$ sample bias for polymer/NC blends (solid lines) and pure polymer (dashed lines). Three separate polymer/NC blends with the same NC concentration are shown to indicate the repeatability of the result. The pump wavelength is $975 \mathrm{~nm}$ allowing selective excitation of the NCs. The inset shows the device structure.

termination of the type of heterostructure. Thus, in addition to demonstrating the potential for device applications, the present work provides insight into the type of heterostructure present.

The PbS nanocrystals were synthesized via the solution phase organometallic approach. ${ }^{22}$ The as-prepared NCs were passivated with a capping layer of oleate ligands, 18-carbon chains, which coordinate to the $\mathrm{Pb}$ sites. In order to provide better charge transfer from $\mathrm{NC}$ to polymer, a postsynthetic ligand exchange was performed to replace the bulky oleates with shorter 8-carbon octylamine ligands. ${ }^{3}$ Octylaminecapped NCs were dispersed in either toluene or chloroform and combined with a solution of MEH-PPV to give a known weight percent. The sample reported here contained $\sim 60 \mathrm{wt} \%$ of NCs. The composite solution was then spincoated onto an ITO slide, forming films of thickness $50-70 \mathrm{~nm} .3 \mathrm{~mm}^{2}$ metal contacts were then deposited by thermal evaporation: a $\sim 100 \mathrm{~nm} \mathrm{Mg}$ layer followed by a $\sim 25 \mathrm{~nm}$ gold capping layer. The final device structure is depicted in the inset to Fig. 2. Samples were stored under a nitrogen environment.

Absorbance spectra (Fig. 1) of NC solutions, pure polymer films, and composite films were obtained using a Cary 500 UV-Vis-NIR spectrophotometer. The spectra confirm that in the long wavelength region beyond the absorption of the polymer, the NCs were responsible for absorption in the polymer/NC blend. The increased absorption of the blend in the 300-400 $\mathrm{nm}$ region, compared to the pure polymer film, is also due to the NCs.

Photoconductivity measurements were performed by measuring, using a lock-in amplifier, the voltage across a $1 \mathrm{k} \Omega$ load resistor in series with the device. The pump source was a $975 \mathrm{~nm}$ fiber-coupled semiconductor laser with adjustable power in the range $0-400 \mathrm{~mW}$. The pump was directly modulated at $50 \mathrm{kHz}$. The $975 \mathrm{~nm}$ pump wavelength allowed selective excitation of the NCs in the polymer/NC composite. Measured photocurrent versus pump intensity at $+5 \mathrm{~V}$ sample bias is presented in Fig. 2. NC/polymer composites are shown as well as pure MEH-PPV control samples. The control samples show negligible response to

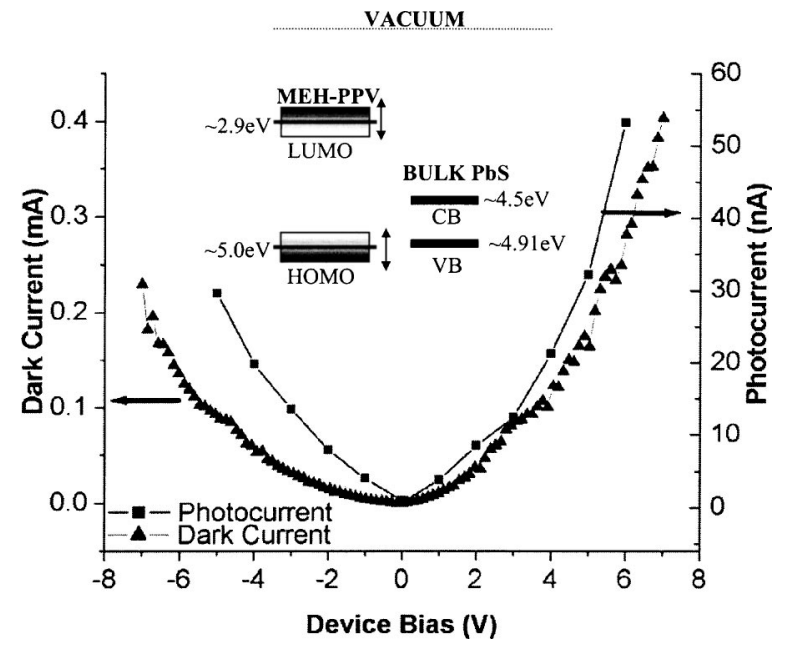

FIG. 3. Absolute values of dark current and photocurrent as a function of sample bias. The inset is a schematic demonstrating the approximate energy levels for MEH-PPV and bulk PbS. The arrows stress the uncertainty in these levels. The schematic also demonstrates the proximity of the HOMO level in the polymer to the valence band in bulk PbS, which adds to the uncertainty in the type of heterostructure present. The energy levels shown for $\mathrm{PbS}$ are for the bulk material, but these levels will shift away from the bulk values in the nanocrystalline form, further complicating the issue of the heterostructure.

the near-infrared pump. In contrast, the composites show significant photocurrent, indicating infrared sensitization of the polymer by the NCs. The response is linear at lower intensities and sublinear at higher intensities, in agreement with other work on related systems, consistent with bimolecular recombination at higher intensities. ${ }^{13}$ The observed photocurrent indicates an internal quantum efficiency (i.e., the number of charges collected at an electrode divided by the number of photons absorbed in the device) of $\sim 5 \times 10^{-6}$ to $\sim 10^{-5}$ charges/photon with a $5 \mathrm{~V}$ bias.

Figure 3 shows the variation in photocurrent with sample bias under $\sim 90 \mathrm{~mW}$ pump power and $50 \mathrm{kHz}$ modulation; also shown is the dark $I-V$ curve. The photocurrent increases with applied bias in a similar manner to the dark current; the asymmetry is due to contact work function offset.

The spectral response of the device was investigated to provide further corroboration that the NCs were acting as a sensitizer for the polymer. A wavelength tunable picosecond laser system operating at a repetition rate of $1 \mathrm{kHz}$ was used as an excitation source. The average power delivered to the sample was $\sim 5 \mathrm{~mW}$ at all wavelengths. The response is shown in Fig. 4. The photocurrent increases with the absorption of the NCs, while negligible response occurs at wavelengths beyond the onset of absorption in the NCs. This provides further evidence that the photocurrent is indeed due to infrared sensitization of the polymer by the NCs.

The same material system, MEH-PPV/PbS-NC, has previously been used to demonstrate electroluminescence. ${ }^{3}$ The demonstration of both electroluminescence and charge separation in the system is consistent with a marginal type-I/ type-II band offset between PbS and MEH-PPV.

In summary, we have presented photoconductivity in the infrared using a conjugated polymer/NC composite. The MEH-PPV/PbS-NC system shows photoconductivity at wavelengths where only the nanocrystal component is absorbing. This photoresponse corresponds to infrared sensitization of the polymer by the NCs. Upon illumination, pho- 


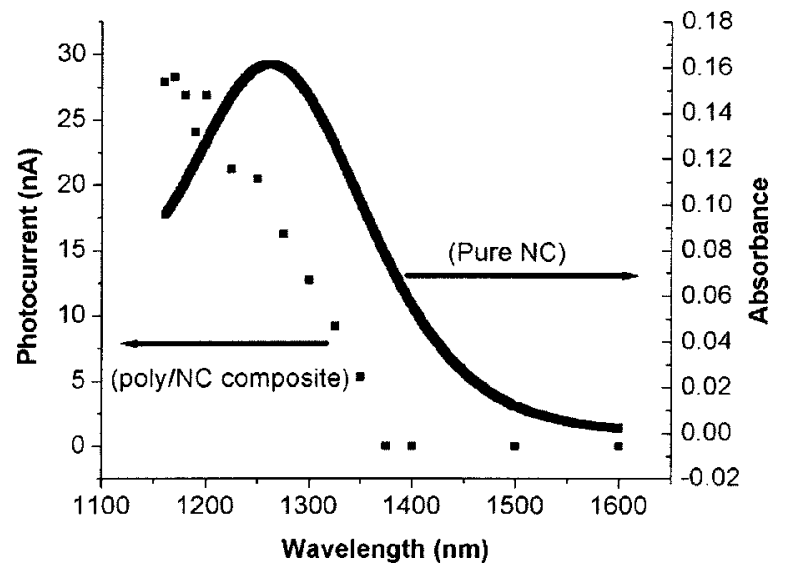

FIG. 4. Photocurrent spectral response of the polymer/NC blend (dots) compared to the absorption of the NCs in solution (line).

togenerated holes in the PbS-NCs are transferred to the MEH-PPV, resulting in photoconductance. The efficiency will be significantly improved by optimization of various parameters such as the ligand barrier, film thickness, NC concentration, and contacts. The principles presented here are general and should allow demonstration of the wavelength tunability of the response by varying the size of the NCs. The results indicate the possibility of using these easily processed nanocomposites as large-area, low-cost, wavelength-tunable photodetectors in the infrared spectral region of interest in communications, sensing, night vision, and biomedical imaging.

The authors gratefully acknowledge the support of Nortel Networks, the Natural Sciences and Engineering Research Council of Canada under the Collaborative Research and Development Program, Materials and Manufacturing Ontario, the Canada Research Chairs Program, the Canada Foundation for Innovation, and the Ontario Innovation Trust, and would like to thank L. Bakueva for many helpful discus- sions and V. Sukhovatkin for assistance in tunable laserbased investigations of the devices reported herein.

${ }^{1}$ S. Coe, W-K. Woo, M. Bawendi, and V. Bulovic, Nature (London) 420, 800 (2002).

${ }^{2}$ J. S. Steckel, S. Coe-Sullivan, V. Bulovic, and M. Bawendi, Adv. Mater. (Weinheim, Ger.) 15, 1862 (2003).

${ }^{3}$ L. Bakueva, S. Musikhin, M. A. Hines, T.-W. F. Chang, M. Tzolov, G. D. Scholes, and E. H. Sargent, Appl. Phys. Lett. 82, 2895 (2003).

${ }^{4}$ N. Tessler, V. Medvedev, M. Kazes, S. Kan, and U. Banin, Science 295, 1506 (2002).

${ }^{5}$ H.-J. Eisler, V. C. Sundar, M. G. Bawendi, M. Walsh, H. I. Smith, and V. Klimov, Appl. Phys. Lett. 80, 4614 (2002).

${ }^{6}$ A. V. Malko, A. A. Mikhailovsky, M. A. Petruska, J. A. Hollingsworth, H. Htoon, M. G. Bawendi, and V. I. Klimov, Appl. Phys. Lett. 81, 1303 (2002).

${ }^{7}$ S. A. Empedocles and M. G. Bawendi, Science 278, 2114 (1997).

${ }^{8}$ V. L. Colvin, K. L. Cunningham, and A. P. Alivisatos, J. Chem. Phys. 101, 7122 (1994).

${ }^{9}$ E. J. D. Klem, L. Levina, and E. H. Sargent (unpublished).

${ }^{10}$ W. U. Huynh, J. J. Dittmer, and A. P. Alivisatos, Science 295, 2425 (2002).

${ }^{11}$ S. Kim, Y. T. Lim, E. G. Soltesz, A. M. De Grand, J. Lee, A. Nakayama, J. A. Parker, T. Mihaljevic, R. G. Laurence, D. M. Dor, L. H. Cohn, M. G. Bawendi, and J. V. Frangioni, Nat. Biotechnol. 22, 93 (2004).

${ }^{12}$ Y. Wang and N. Herron, Chem. Phys. Lett. 200, 71 (1992).

${ }^{13}$ N. C. Greenham, X. Peng, and A. P. Alivisatos, Phys. Rev. B 54, 17628 (1996).

${ }^{14}$ D. S. Ginger and N. C. Greenham, Phys. Rev. B 59, 10622 (1999).

${ }^{15}$ D. S. Ginger and N. C. Greenham, J. Appl. Phys. 87, 1361 (2000).

${ }^{16}$ C. A. Leatherdale, C. R. Kagan, N. Y. Morgan, S. A. Empedocles, M. A. Kastner, and M. G. Bawendi, Phys. Rev. B 62, 2669 (2000).

${ }^{17}$ N. Y. Morgan, C. A. Leatherdale, M. Drndic, M. V. Jarosz, M. A. Kastner, and M. G. Bawendi, Phys. Rev. B 66, 075339 (2002).

${ }^{18}$ P. Hoyer and R. Konenkamp, Appl. Phys. Lett. 66, 349 (1995).

${ }^{19}$ J. G. Winiarz, L. Zhang, J. Park, and P. N. Prasad, J. Phys. Chem. 106, 967 (2002).

${ }^{20}$ Y. Greenwald, X. Xu, M. Fourmigue, G. Srdanov, C. Koss, F. Wudl, and A. J. Heeger, J. Polym. Sci., Part A: Polym. Chem. 36, 3115 (1998).

${ }^{21}$ S-H. Jin, M-S. Jang, H-S. Suh, H-N. Cho, J-H. Lee, and Y-S. Gal, Chem. Mater. 14, 643 (2002).

${ }^{22}$ M. A. Hines and G. D. Scholes, Adv. Mater. (Weinheim, Ger.) 15, 1844 (2003). 